\title{
CURCUMIN FOR ALZHEIMER'S DISEASE (AD) POTENTIAL TREATMENT
}

\author{
Dias Rima Sutiono* and Steven Iasmartua \\ Bio Medicine Study Program, Institut Bio Scientia Internasional Indonesia (Indonesia International Institute for \\ Life Sciences, i3L) \\ * Corresponding Email : sutiono.dr@gmail.com
}

\begin{abstract}
Various studies had been conducted regarding the effect of curcumin on AD patients, thus, many of the studies had suggested that curcumin had the potential to prevent and treat $A D$ through several molecular mechanisms including act as anti-inflammatory, anti-oxidant, binding the $A \beta$ plaques, metal chelation, and lowering cholesterol level. One of the prominent characteristics of this neurodegenerative disease is shown by the presence of beta amyloids plaques $(A \beta)$ and inflammation inside the patient's brains; and as mention above curcumin had shown its capabilities in inhibiting the $A \beta$ plaques and act as an anti-inflammation agent.
\end{abstract}

Keywords: Alzheimer's disease, curcumin, beta amyloid $(A \beta)$ plaques, neuroinflammation, anti-inflammation.

\section{ALZHEIMER'S DISEASE}

Alzheimer's disease $(\mathrm{AD})$ is a neurodegenerative disease and the most common form of dementia. According to World Alzheimer Report $2015^{9}$ statistically the dementia prevalence in people aged 60 years and above is estimated roughly to range from 10\% in North and South America, 8.7\% in North Africa and Middle East, 4.6\% in Central Europe, and 7.6\% in Asia. In 2015 a total number of 46.8 million people worldwide are living with dementia with $\mathrm{AD}$ contribute for $11 \%$ of the cases. Alzheimer disease International also speculate by referring to the world trend statistics that the number of dementia will almost double every 20 years, it will reach 74.7 million people in 2030, and 131.5 million people in 2050. Moreover, the estimated rate of dementia keeps increasing along the time when comparing the rate between World Alzheimer Report 2015 and 2009.

$\mathrm{AD}$ patient characterized with progressive cognitive deterioration along with declining psychological and physical condition such as problems in language, disorientation, mood swings, behavioral issues, and gradual body function lost. Moreover, if the disease keeps progressing it will eventually lead to death due to the large amount of neurological damage inside the patient's brain making the body not functional as normal condition ${ }^{12}$.

Currently the particular cause of $\mathrm{AD}$ is still unknown, but it is suggested several risk factors that could potentially lead to $\mathrm{AD}$ including age (60 years above), genetics, head injury, metabolism disease (diabetes, high 
cholesterol), depression and hypertension ${ }^{16}$. Current treatment also not been found yet, but some therapies may relief and slowing the progression rate of $\mathrm{AD}$. Although, the specific cause and curing treatment is elusive the study of neuropathology of $\mathrm{AD}$ had been done in many years and new information still retrieved along with the development of medical technologies.

Therefore, scientist tries to find the cure for $\mathrm{AD}$ in many ways, from high-tech medical treatment to alternative medicine such as herbal. In regards to alternative medicine, scientists had natural compound derived from turmeric that may be have the potential to cure $\mathrm{AD}$ called curcumin; it is also believed to have many medicinal properties ${ }^{2}$. It is also beneficial in term of natural products, accessibility, and price particularly for patient from middle-low class economy.

\section{CURCUMIN}

Curcumin is a bioactive compound derived from a rhizome of a species of plant named Curcuma longa or commonly known as turmeric (a native plant from South East Asia region). It was first classified in 1815 by Vogel and Pelletier, later on the crystalline form was obtained in 187. For centuries turmeric had been used primarily as a food additive in many cuisines including curry, opor, and other spicy dishes; also in Asia it was believed and used as a natural herbal remedies to treat common symptoms (fever, inflammation, rash, bug bite, etc.). Currently scientist had found that curcumin have a medicinal and other health benefits, thus, varieties of products based on curcumin as the main component start to emerge ${ }^{3}$.

Nowadays, curcumin commonly found in the form of powder with a bright-yellow color. It was identifying as phytopolyphenol, the structure of curcumin is mainly composed of carbon chain linking with two aryl groups. When ingested by human, curcumin can distribute inside the blood with a half-life of approximately 8 hours; curcumin shown sensitivity towards several factors including visible light, ultraviolet light, high $\mathrm{pH}$ level, as well as oxygen ${ }^{16}$.

Scientists currently had conducting many research regarding to the curcumin medical properties, it is significantly proven to have antioxidant properties which had been shown in the chemical structure of the curcumin itself located in the phenolic $(\mathrm{OH})$ groups that attached with the aryl group capable to reducing free radicals such as ROS (reactive oxygen species) ${ }^{8}$. Therefore, the number of research over curcumin had increase and many of medical application of curcumin had been suggested including inhibits cancer cells, metal chelation, and AD treatment.

\section{EFFECTS OF CURCUMIN ON AD}

A various scientific studies have shown the potential of curcumin towards mechanism of Alzheimer's disease treatment. As a neurodegenerative disease $\mathrm{AD}$ major infection occur inside the brain, thus, it is important to observe the connection between $\mathrm{AD}$ and curcumin particularly on the blood-brain-barrier (BBB) which is a highly selective semi permeable barrier (allows some molecules such as water, some gases, and lipid soluble molecules by passive diffusion) that protects and separates the brain and spinal cord blood circulation from the rest of the body ${ }^{16}$. Based on research conducted by Ringman and colleagues in 2012 curcumin was shown to have the capabilities to cross the BBB membrane due to its hydrophobicity and lipophilic nature. Therefore, it indicates that curcumin medical properties can be applied inside the $\mathrm{AD}$ patient's brain. 


\section{INHIBITION OF BETA AMYLOID PLAQUES BY CURCUMIN}

The formations of beta-amyloid $(\mathrm{A} \beta)$ plaques along with the presence of neurofibrillary tangle are the one of the prominent characteristics shown in $\mathrm{AD}$ patient's brain ${ }^{5}$. In normal condition, amyloid precursor protein (an integral membrane protein) or so called APP is sectioned by multiple enzymes including $\alpha$-secretase and $\gamma$-secretase and release $\mathrm{p} 3$ peptides which is degrade (the function towards brain is still elusive until now) ${ }^{15}$. However, in $\mathrm{AD}$ the APP is cleaved with $\beta$-secretase instead of $\alpha$-secretase, thus, it cleaved on different site by $\gamma$-secretase resulting betaamyloid $(A \beta) d$ peptide that cannot be regulated. $A \beta$ protein is binding to another $A \beta$ resulting $A \beta$ plaques that can trigger neurotoxic effects and capable to degrade brain cells by tau hyper phosphorylation ${ }^{16}$.

Correspond to $\mathrm{AD}$ prominent characteristics, currently the development of $\mathrm{AD}$ treatment has been targeting the $\mathrm{A} \beta$ plaques including pharmacological treatments, immunotherapies, and medicinal herbals. From the previous studies some substances including ibuprofen and Congo red known to bind to amyloid fibril and shown to reduce the accumulation of $\mathrm{A} \beta$ plaque; however, both are poor brain penetrant and could cause toxicity given in chronic dose $^{7,8}$.

Several studies had demonstrated the potential effect of curcumin on the inhibition of $A \beta$ plaques. According to the experiment conducted by team of scientists led by Giselle P. Lim in 2001, they observed the effect of high $(5000 \mathrm{ppm})$ and low $(160 \mathrm{ppm})$ level dose of curcumin towards APP and A $\beta$ plaques of a mice. The statics shown mice given with low dose of curcumin significantly reduce the amount of insoluble $A \beta$, and soluble $A \beta$ (p3 peptide), thus, decreased the plaque burden by $43-50 \%$. Notably, somehow the mice given high dose of curcumin did not show significant reduced number; also the levels of APP presence on the membrane were not reduced. Further research done by Chi-Fen Lin and colleagues in 2013, had shown similar results regarding the decreasing number of $A \beta$ plaques in mice when given with low dose of curcumin $(50 \mu \mathrm{M})$.

\section{CURCUMIN AS ANTI-INFLAMMATION AND MICROGLIAL ACTIVATION}

Aside from $\mathrm{A} \beta$ plaques, inflammation on the brain also occurs in $\mathrm{AD}$ patients. However, this occurrence is different with the inflammation in general term, because it does not show fever, swelling, redness, and pain as it supposed to be. Therefore, this type of inflammation called neuroinflammation; instead of showing the general symptoms it causes damage to neurological functions ${ }^{13}$. Neuroinflammation is caused by immune mechanism of the microglia cells (macrophage-like cells inside brain) generating constant number of proinflammatory cytokine eventually causing neuronal death. Microglia triggers inflammation as well maintaining its function as phagocytes that engulf $\mathrm{A} \beta$ plaques and repair damaged brain cells ${ }^{12}$. Although, in $\mathrm{AD}$ the microglia was somehow deceived, thus, it activated mostly to proliferate rather than engulf the $A \beta$ plaques ${ }^{4}$.

In an experiment conducted by Milan Fiala and team in 2007 on investigating the effect of curcumin towards innate immunity response inside the $\mathrm{AD}$ patients brain, it was first hypothesized that curcumin may enhance innate immune system and increase the clearance of $A \beta$ plaques. They observed the microglia activities through isolated macrophage cultures technique combine with the tissue derived from frozen frontal lobe section infected by $\mathrm{AD}$ to obtain the $A \beta$ plaques; it was incubated and stained by indirect immunofluorescence (conjugate secondary antibodies CD68). Then it was added with isolated and purified curcumin inside the macrophage cultures in vitro. The experiments shown the curcumin enhance the microglia phagocytosis activity of $A \beta$ plaques in $50 \%$ of the cases out of 48 samples. Further measurement of the curcumin activity was recorded through phagocytosis assay 
using fluorescence and confocal microscopy and the sample which added curcumin shown significance activity of phagocytosis compare to the control samples ${ }^{5}$.

In the 24th Annual Southern California Alzheimer's Disease Research Conference September 20, 2013; Andrea Tenner, Ph.D. stated in her presentation that microglia play an important role in $\mathrm{AD}$ it act both induce inflammation and phagocytosis the $\mathrm{A} \beta$ plaques as mention before; she also stated that the action in microglia could actually controlled by several factors, thus it is possible to breakdown each function of the microglia itself and regulate the desire microglia activation. Researcher from University of Southern California Los Angeles (UCLA) had conducted a study particularly in curcumin inhibition activities of microglial proliferation and differentiation ${ }^{1}$ The observation was done by giving a gradual doses of curcumin $(4,5,10,15,20 \mu \mathrm{M})$ on AD mice, after a six-day trial it shown that curcumin inhibit the proliferation of microglia cells, by undergo differentiation and maturation eventually cell apoptosis. It was also found that curcumin inhibits not only microglia but neuroglia proliferation. The inhibition of curcumin seems to be dose dependently; it was observed that the higher curcumin concentration given the greater the inhibition. In short, curcumin involves in inhibition of neuroglia proliferation and improving the microglia $A \beta$ plaques phagocytosis ${ }^{11,14}$.

\section{CONCLUSION}

Based on the data and discoveries from studies that had mention above, curcumin may become the future potent treatment for curing and preventing AD. Particularly in their properties to inhibit the production of $A \beta$ plaques in $\mathrm{AD}$ patients and enhance the microglia cell activation by reducing the neuroinflammation occur in infected brain. However, due to many of the studies and experiment conducted on in vitro and animals based experiments, further research had to be conducted to confirm it full function in larger organisms such as human. It also unclears on how the drug administration of curcumin can be effective either by taking it directly (orally, intravenous, etc.) or indirectly through cuisines (as food additives and spices). But by doing so, a new curcumin based product may be generated with the advantages on accessibility, economical achievable, and practical administration to cure neurodegenerative disease specifically Alzheimer's disease.

\section{REFERENCE}

Ambegaokar, S. S., Wu, L., Alamshahi, K., Lau, J., Jazayeri, L., Chan, S., ... Timiras, P. S. (2003). Curcumin Inhibits Dose-dependently and Time-dependently Neuroglial Cell Proliferation and Growth, (JANUARY 2004).

Basnet, P., \& Skalko-Basnet, N. (2011). Curcumin: An anti-inflammatory molecule from a curry spice on the path to cancer treatment. Molecules, 16(6), 4567-4598. doi:10.3390/molecules 16064567

Bhowmik, D., Kumar, K. P., Chandira, M., Jayakar, B., \& Jit. (2009). Turmeric: A Herbal and Traditional Medicine. Archives of Applied Science Research, 1(2), 86-108.

Doens, D., \& Fernández, P. L. (2014). Microglia receptors and their implications in the response to amyloid $\beta$ for Alzheimer's disease pathogenesis. Journal of Neuroinflammation, 11, 48. doi:10.1186/1742-2094-11-48

Fiala, M., Liu, P. T., Espinosa-Jeffrey, A., Rosenthal, M. J., Bernard, G., Ringman, J. M., ... Cashman, J. (2007). Innate immunity and transcription of MGAT-III and Toll-like receptors in Alzheimer's disease patients are improved by bisdemethoxycurcumin. Proceedings of the National Academy of Sciences of the United States of America, 104(31), 12849-12854. doi:10.1073/pnas.0701267104 
Lim, G. P., Chu, T., Yang, F., Beech, W., Frautschy, S. a, \& Cole, G. M. (2001). The curry spice curcumin reduces oxidative damage and amyloid pathology in an Alzheimer transgenic mouse. The Journal of Neuroscience : The Official Journal of the Society for Neuroscience, 21(21), 8370-8377. doi:21/21/8370 [pii]

Lim, G. P., Yang, F., Chu, T., Gahtan, E., Ubeda, O., Beech, W., ... Cole, G. M. (2001). Ibuprofen effects on Alzheimer pathology and open field activity in APPsw transgenic mice. Neurobiology of Aging, 22(6), 983-991. doi:10.1016/S0197-4580(01)00299-8

Lin, C.-F., Yu, K.-H., Jheng, C.-P., Chung, R., \& Lee, C.-I. (2013). Curcumin Reduces Amyloid Fibrillation of Prion Protein and Decreases Reactive Oxidative Stress. Pathogens, 2(3), 506-519. doi:10.3390/ pathogens 2030506

Prince, M., Wimo, A., Guerchet, M., Gemma-Claire, A., Wu, Y.-T., \& Prina, M. (2015). World Alzheimer Report 2015: The Global Impact of Dementia - An analysis of prevalence, incidence, cost and trends. Alzheimer's Disease International, 84. doi:10.1111/j.0963-7214.2004.00293.x

Ravindran, P., Babu, K., \& Sivaraman, K. (2007). Turmeric: the genus Curcuma.

Ringman, J. M., Frautschy, S. a, Teng, E., Begum, A. N., Bardens, J., Beigi, M., ... Cole, G. M. (2012). Oral curcumin for Alzheimer's disease: tolerability and efficacy in a 24-week randomized, double blind, placebo-controlled study. Alzheimer's Research \& Therapy, 4(5), 43. doi:10.1186/alzrt146

Ringman, J. M., Frautschy, S. A., Cole, G. M., Masterman, D. L., \& Cummings, J. L. (2005). A potential role of the curry spice curcumin in Alzheimer's disease. Current Alzheimer Research, 2(2), 131-6. doi:10.2174/1567205053585882

Tenner, A. (2013). Inflammation as a Therapeutic Target in Alzheimer's Disease.

Tizabi, Y., Hurley, L. L., Qualls, Z., \& Akinfiresoye, L. (2014). Relevance of the anti-inflammatory properties of curcumin in neurodegenerative diseases and depression. Molecules, 19(12), 20864-80879. doi:10.3390/ molecules 191220864

Yang, F., Lim, G. P., Begum, A. N., Ubeda, O. J., Simmons, M. R., Ambegaokar, S. S., ... Cole, G. M. (2005). Curcumin inhibits formation of amyloid oligomers and fibrils, binds plaques, and reduces amyloid in vivo. Journal of Biological Chemistry, 280(7), 5892-5901. doi:10.1074/jbc.M404751200

Yao, E. C., \& Xue, L. (2014). Therapeutic Effects of Curcumin on Alzheimer's Disease, (December), 145-159. 